\title{
THE NUMBER OF SOLUTIONS TO THE CLASSICAL PLATEAU PROBLEM IS GENERICALLY FINITE
}

\author{
BY R. BÖHME AND A. J. TROMBA
}

Communicated by S. S. Chern, January 21, 1977

0. Introduction. The question of how many solutions there are to the classical problem of Plateau has been open for roughly a century. Existence of at least one solution was proved in 1931 independently by $\mathrm{T}$. Rado [7] and J. Douglas [3]. Courant, in his book Dirichlet's principle, conformal mappings and minimal surfaces [2], outlines an argument which suggests that there may exist rectifiable curves in $\mathbf{R}^{\mathbf{3}}$ bounding on uncountable number of solutions. It has been believed for some time that for all sufficiently nice curves there are only a finite number of surfaces of mean curvature zero which they bound. In this note we state that there exists an open dense set of curves which bound a finite number of classical minimal surfaces of the type of the two disc. This result essentially is a synthesis of the ideas of [1], [9], [10] .

I. Formulation of results. Let $\alpha: S^{1} \rightarrow \mathbf{R}^{n}$ be a $C^{\infty}$ embedding of $S^{1}$ into $\mathrm{R}^{n}$, where $S^{1}$ denotes the boundary of the open disc $D$ in $\mathbf{R}^{2}$. Let $\Gamma^{\alpha}=$ $\alpha\left(S^{1}\right)$ denote its image.

Definition. A classical solution to Plateau's problem for $\alpha$ is a map $u$ from $\bar{D}$ into $\mathbf{R}^{n}$ satisfying the following properties.

(i) $u \in C^{0}(\bar{D}) \cap C^{\infty}(D)$,

(ii) $\Delta u=0$,

(iii) $\partial u / \partial x \cdot \partial u / \partial y=0 \forall(x, y) \in D$,

(iv) $\|\partial u / \partial x\|=\|\partial u / \partial y\| \forall(x, y) \in D$,

(v) $u: S^{1} \rightarrow \Gamma^{\alpha}$ homeomorphically.

REMARK.1. By well-known regularity results for minimal surfaces first proved by Hildebrandt [5], and then later improved by Nitsche [6], Heinz and Tomi [4], and Tomi [8], $\alpha \in C^{\infty}$ implies $u \in C^{\infty}(\bar{D})$.

Let $\mathrm{A}$ denote the space of all $C^{\infty}$ embeddings of $S^{1}$ into $\mathbf{R}^{n}$ with the $C^{\infty}$ topology.

THEOREM 1. There exists an open and dense subset $A_{0} \in A$ such that for all $\alpha \in A_{0}$ there are only finitely many classical solutions of the Plateau problem.

REMARK 2. These finitely many surfaces are nondegenerate in the sense described in [1].

AMS (MOS) subject classifications (1970). Primary 53A10, 65N99, 58E99. 
REMARK 3. There is an $H^{k}$ Sobolev space version of Theorem 1. As a direct corollary of this fact we obtain that for $\alpha \in A_{0}$ the set of solutions are differentiable functions of $\alpha$. This immediately implies the stability of the number of solutions in $A_{0}$.

REMARK 4. The equations in the above definition are invariant under the action of the conformal group of $D$. In Theorem 1 surfaces equivalent under the action of this group are identified.

DEFinition. A branch point $p \in \bar{D}$ of a minimal surface $u$ is a point where $u$ fails to be an immersion.

THEOREM 2. If $\alpha \in A_{0}$ it bounds no minimal surface with branch points on the boundary, and if $n>3$ it bounds no minimal surface with boundary or interior branch points.

\section{REFERENCES}

1. R. Böhme, Stability of minimal surfaces, Sympos. on Function Theoretic Methods for Partial Differential Equations (Darmstadt, 1976), Lecture Notes in Math. (to appear).

2. R. Courant, Dirichlet's principle, conformal mappings, and minimal surfaces, Interscience, New York, 1950. MR 12, 90.

3. J. Douglas, Solution of the problem of Plateau, Trans. Amer. Math. Soc. 33 (1931), $263-321$.

4. E. Heinz and F. Tomi, Zu einem Satz von Hildebrandt über das Randverhalten von Minimalflächen, Math. Z. 111 (1969), 372-386. MR 42 \#975.

5. S. Hildebrandt, Boundary behavior of minimal surfaces, Arch. Rational Mech. Anal. 35 (1969), 47-82. MR 40 \#1901.

6. J. C. C. Nitsche, The boundary behavior of minimal surfaces. Kellog's theorem and branch points on the boundary, Invent, Math. 8 (1969), 313-333. MR 41 \#4399a. lin, 1933.

7. T. Rado, On the problem of Plateau, Ergebnisse Math. Grenzgebiete, Springer, Ber-

8. F. Tomi, Ein einfacher Beweis eines Regularitätssatzes für schwache Lösungen gewisser elliptischer Systeme, Math. Z. 112 (1969), 214-218. MR 41 \#2199.

9. A. J. Tromba, On the number of solutions to Plateau's problem, Bull. Amer. Math. Soc. 82 (1976), 66-68.

10. A. J. Tromba, On the number of simply connected minimal surfaces spanning a curve, Mem. Amer. Math. Soc. (to appear). GERMANY

DEPARTMENT OF MATHEMATICS, UNIVERSITY OF ERLANGEN, NÜRNBERG,

DEPARTMENT OF MATHEMATICS, UNIVERSITY OF CALIFORNIA, SANTA CRUZ, SANTA CRUZ, CALIFORNIA 95064 